Ann. Biol. anim. Bioch. Biophys., 1973, 13 (3), 505-519.

\title{
CONSTRUCTION ET FONCTIONNEMENT DE 4 CHAMBRES RESPIRATOIRES DU TYPE « CIRCUIT FERMÉ » POUR DES ÉTUDES DE NUTRITION ÉNERGÉTIQUE CHEZ LE RAT EN CROISSANCE
}

\author{
M. VERMOREL, J.-C. BOUVIER et Y. BONNET \\ Station d'Étude des Métabolismes, \\ Centre de Recherches de Clermont Ferrand, I. N. R. A., \\ Saint Genès Champanelle, 63110 Beaumont
}

\section{RÉSUMÉ}

4 chambres respiratoires du type "circuit fermé " ont été conçues pour des études de nutrition énergétique chez le Rat en croissance. Ces installations permettent de réaliser des bilans énergétiques et azotés précis sur une période de 24 heures, au minimum. Le gaz carbonique est capté par des tamis moléculaires $5 \AA$ et la quantité produite est déterminée par gravimétrie. L'oxygène est fourni soit par un spiromètre, soit un dispositif d'alimentation automatique, avec détermination gravimétrique de la quantité d'oxygène délivré. Les bilans énergétiques et azotés. sont réalisés sur une période de 5 jours avec des sujets de même poids ; la précision des différentes. mesures ainsi que la concordance entre les 2 types de bilans réalisés montrent que la méthode utilisée est satisfaisante pour les études envisagées.

\section{I. - INTRODUCTION}

Un des thèmes de recherche poursuivis à la Station d'Étude des Métabolismes est l'étude de la valeur énergétique de certains nutriments pour les animaux en croissance et de leur influence sur l'orientation du métabolisme : accroissement de la protéinogenèse et de la lipogenèse. Le Rat blanc est utilisé comme animal expérimental pour préparer les études réalisées ensuite sur Agneau, Veau ou Porcelet.

Les expérimentations en lots par la méthode des abattages comparés nous fournissent des bilans globaux précis (énergie, azote, acides gras...) sur une période d'assez longue durée ( 3 à 4 semaines) qui recouvre des stades physiologiques diffé- 
rents (du sevrage, vers $70 \mathrm{~g}$, à une phase postpubertaire, 200 à $240 \mathrm{~g}$ ). En complément de cette méthode nous réalisons des bilans énergétiques et azotés sur des rats pris individuellement à plusieurs stades physiologiques au cours de leur croissance : après le sevrage (70 à Ioo g), avant la puberté (r50 à I $80 \mathrm{~g}$ ), après la puberté (230 à $260 \mathrm{~g})$.

Pour ces études nous avons construit et nous utilisons depuis plusieurs années 4 chambres respiratoires, du type circuit fermé qui, par leur conception, répondent aux impératifs expérimentaux : mesures pendant 24 heures, plusieurs jours consécutifs, confort des rats permettant des croissances rapides, séparation et collecte parfaites des fèces et de l'urine, détermination précise des quantités d'oxygène consommé et de gaz carbonique produit et du quotient respiratoire. Ces échanges respiratoires permettent de calculer la production de chaleur $(Q)$ de l'animal et, par différence, de déterminer la quantité d'énergie (E) fixée :

$$
\mathrm{E} \text { fixée }=\mathrm{E} \text { ingérée }-\mathrm{E} \text { fèces }-\mathrm{E} \text { urine }-\mathrm{Q}
$$

\section{II. - DESCRIPTION DES INSTALLATIONS}

Avec l'expérience du fonctionnement des 2 premières chambres respiratoires nous avons apporté certaines modifications dans la réalisation des 2 suivantes :

\section{A. - La cage à digestibilité}

Le rat est placé dans une cage métallique, grillagée, cylindrique, de $17 \mathrm{~cm}$ de diamètre et de hauteur, disposée sur un bâti mobile (fig. I). La ration est distribuée sous forme de pâtée fluide dans une mangeoire placée à l'extérieur, très accessible par le rat et évitant tout gaspillage. L'abreuvoir est constitué d'un flacon renversé muni d'une tubulure métallique.

Le dispositif de séparation des fèces et de l'urine est un entonnoir en pyrex modifié à sa base (fig. 2). Les fèces roulent sur les parois et tombent dans un cristallisoir placé sous l'orifice. L'urine ruisselle le long des parois de l'entonnoir, suit la gouttière inférieure et s'écoule dans un bécher contenant quelques gouttes d'acide sulfurique dilué $(4 \mathrm{~N})$. Avec des rats mâles nous n'avons jamais observé de traces d'urine dans les fèces et la présence d'une plaque de protection s'est révélée inutile. Chaque matin les fèces et l'urine sont récoltées et l'entonnoir est rincé avec une pissette.

\section{B. - Les chambres respiratoires}

Les dimensions de la cage à digestibilité ont obligé à construire une chambre respiratoire de grande taille dont le volume (environ 40 l) est compatible avec des mesures sur une période de 24 heures. Cette chambre est un parallélépipède rectangle $(32 \times 26 \times 47 \mathrm{~cm})$ en polychlorure de vinyle transparent, plié et soudé. Les 2 premiers modèles comportent des tubulures latérales soudées pour la circulation de l'air et un couvercle au niveau duquel l'étanchéité est assurée par un joint d'eau.

En raison des risques de fuites d'air au niveau de ces tubulures nous avons préféré pour les 2 chambres suivantes les placer à la partie inférieure des chambres, immergées dans l'eau, le joint d'eau étant également à la base des chambres (fig. 3).

\section{C. - Le circuit de l'air}

Une pompe étanche assure en permanence la circulation de l'air enfermé dans la chambre respiratoire à travers un train d'absorbeurs d'eau et de gaz carbonique. La diminution de pression correspondante provoque une arrivée d'oxygène en remplacement de celui qui a été consommé par l'animal. 

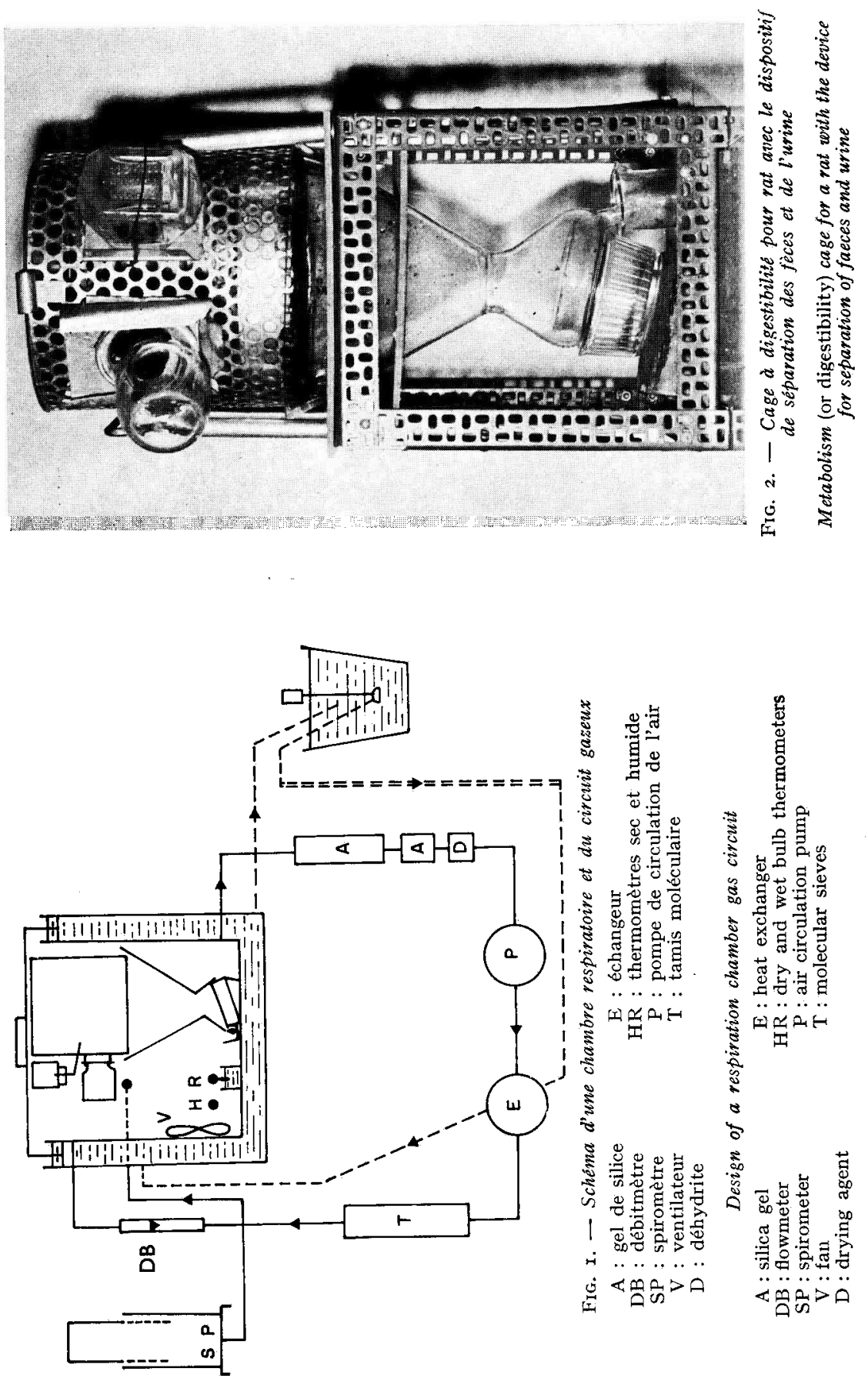


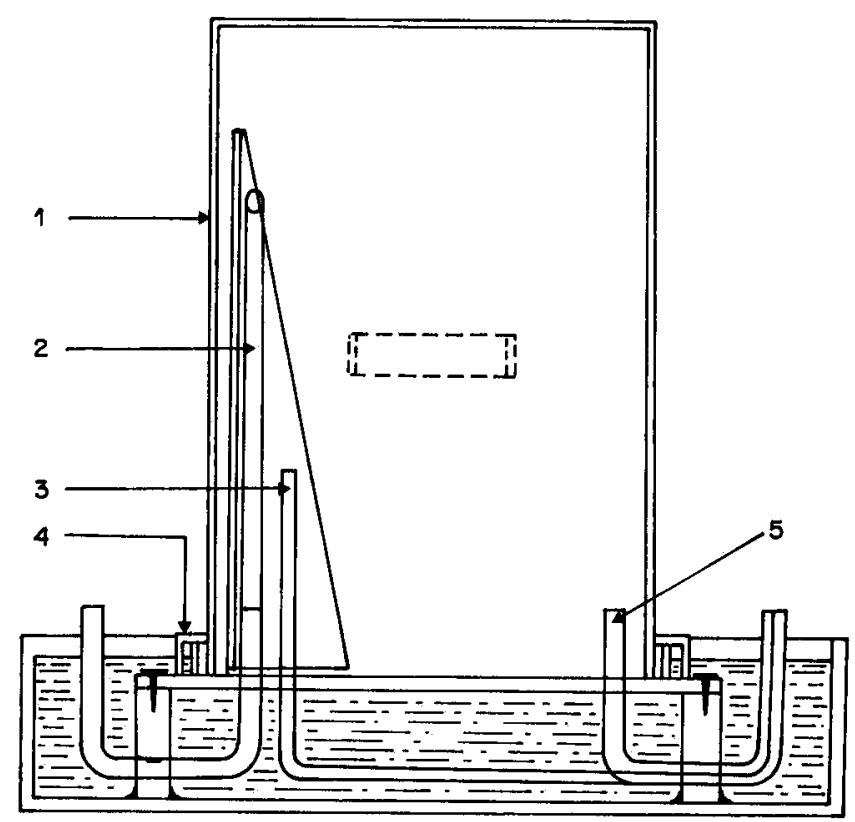

FIG. 3. - Schéma du second modèle de chambre respiratoire, avec joint d'eau à la base I : cloche amovible $(310 \times 250 \times 470 \mathrm{~mm})$ en PVC transparent 2 : entrée d'air $\quad 4:$ joint d'eau

Scheme of the second model of the respiration chamber with a water seal at the base I : Removable hood ( 3 IO $\times 250 \times 470 \mathrm{~mm})$ in transparent $\mathrm{PVC}$

2 : Air inlet

3: Oxygen inlet 4: Water seal 5: Air outlet

\section{Les pompes.}

La circulation de l'air est assurée par l'un des 2 modèles de pompes étanches suivants :

- une pompe XBP 30 (Wauquier-Vanton, Lille) dans laquelle l'air est comprimé entre le corps de la pompe et un flexible en néoprène par un excentrique,

- une pompe Wab (Prolabo) actionnée par un moteur de Ioo watts, I $400 \mathrm{tr} / \mathrm{mn}$; l'air circule à travers un tuyau en rhodorsyl RP 60 (1o $\times$ 13,5) écrasé par les galets de la pompe. Ce tuyau doit être déplacé tous les 2 jours pour éviter les fuites.

Le débit dépend des pertes de charge dans les absorbeurs. Il est mesuré grossièrement à l'aide d'un rotamètre et réglé au moyen de pinces de Mohr suivant la taille et la vitesse de croissance du rat, de façon à maintenir la concentration en gaz carbonique entre o, 3 et 0,5 p. Ioo à l'intérieur de la chambre respiratoire.

\section{La déshydratation de l'air.}

L'air traverse 2 absorbeurs contenant chacun environ $800 \mathrm{~g}$ de gel de silice coloré. Le premier est changé chaque jour, le second chaque semaine, ou plus fréquemment si nécessaire. L'air qui comporte encore des traces d'eau circule à travers 2 absorbeurs contenant environ Ioo $\mathrm{g}$ de déhydrite granulée (perchlorate de magnésium anhydre) (TRussel et DiEHL, 1963).

\section{D. - Mesure de la production de gaz carbonique}

L'air complètement déshydraté traverse un absorbeur en aluminium contenant environ 7oo $g$ de tamis moléculaires de Linde $5 \AA$ (silicoaluminate de sodium et de potassium) qui captent le gaz carbonique par absorption (BoIteau et DeBrock, I96I). Ce produit peut être regénéré par chauffage à $25^{\circ} \mathrm{C}$, ce qui permet de l'utiliser pendant plusieurs années. Malheureusement il est 
très hygroscopique et l'air doit être parfaitement desséché au préalable, ce qui explique l'emploi de la déhydrite. Dans nos conditions expérimentales (température de $2 \mathrm{I}^{\circ} \mathrm{C}$ ), les tamis moléculaires peuvent capter 3 à 4 p. Ioo de leur poids en gaz carbonique sans inconvénient; au-delà de ces valeurs, le gaz carbonique n'est plus piégé complètement et sa concentration dans la chambre respiratoire augmente lentement.

L'absorbeur de $\mathrm{CO}_{2}$ est pesé sur un trébuchet de I ooo $\mathrm{g}( \pm 0,02 \mathrm{~g})$ réglé avant chaque mesure avec une tare de $800 \mathrm{~g}$. La détermination de la quantité de $\mathrm{CO}_{2}$ capté est faite avec une précision de 0,2 à 0,4 p. 100 .

En cours de période de mesure il s'établit un équilibre entre le $\mathrm{CO}_{2}$ produit par le rat et le $\mathrm{CO}_{2}$ capté et la concentration dans la chambre est comprise entre o,3 et $0,6 \mathrm{p}$. roo. Pour tenir compte de cette accumulation de gaz carbonique on prélève un échantillon d'air en début de journée et 2 en fin de journée dans des ampoules en pyrex, sous vide. La teneur en $\mathrm{CO}_{2}$ de l'air est déterminée soit par une méthode volumétrique à l'aide d'un appareil de Carpenter et $\mathrm{Hal}$ dane (absorption du $\mathrm{CO}_{2}$ par une solution de potasse), comparable à celui décrit par SchIEMANN (1957), soit par une méthode physique à l'aide d'un analyseur à rayonnement infrarouge "Uras 2 " o à I p. Ioo (Hartmann-Braun). La quantité de $\mathrm{CO}_{2}$ accumulé dans la chambre respiratoire correspond environ à 2 p. Ioo du $\mathrm{CO}_{2}$ produit par le rat.

\section{E. - Mesure de la consommation d'oxygène}

L'oxygène est fourni à l'animal par 2 procédés légèrement différents, le premier pour les 2 premières chambres construites, le second pour les 2 dernières.

I. Un spiromètre dont la cloche en cuivre a un volume utile de $91: \mathrm{I} 7 \mathrm{~cm}$ de diamètre intérieur et $40 \mathrm{~cm}$ de hauteur utile. Le contrepoids creux est en liaison avec le spiromètre par un tuyau souple, de façon que, lorsque la cloche se vide, la masse du contrepoids diminue d'une quantité égale à la poussée d'Archimède. La cloche est en très légère surpression ( +3 à $+6 \mathrm{~mm}$ d'eau) et baisse pour une diminution de pression de 1 ou $2 \mathrm{~mm}$ d'eau dans la chambre respiratoire; en revanche, elle remonte plus difficilement, ce qui est un inconvénient lors des contrôles d'étanchéité.

La cloche du spiromètre est munie d'une réglette verticale graduée au $1 / 2 \mathrm{~mm}$; les lectures sont faites à l'aide d'une lunette, en particulier pour éviter les erreurs de parallaxe. Son volume a été déterminé sur toute sa hauteur par gravimétrie : la cloche, en place, a été remplie d'eau puis vidée litre par litre avec lectures et pesées précises. L'erreur relative dans la détermination volumétrique de la quantité d'oxygène fournie par la cloche est inférieure ou égale à 0,5 p. Ioo.

2. Une alimentation automatique : l'oxygène est chassé d'un réservoir (A) par de l'eau sous le contrôle d'un manostat (fig. 4 et 5). Une diminution de pression de 1 à $2 \mathrm{~mm}$ d'eau dans la chambre respiratoire, détectée par le manomètre à eau (M) muni d'électrodes, provoque l'ouverture de l'électrovanne EV I commandée par un relais sensible. Cette électrovanne laisse couler dans le réservoir d'oxygène $(A)$ de l'eau contenue dans le récipient $B$ jusqu'à rétablissement de la pression dans la chambre. La mesure de la quantité d'oxygène ainsi délivrée au rat se fait par pesée du récipient $B$ (balance de $15 \mathrm{~kg}$ graduée en $\mathrm{g}$ ), mais il y a lieu d'apporter une correction pour tenir compte de l'influence des variations de température et de pression sur l'oxygène du réservoir $A$. I'erreur relative maximale de cette mesure est de I p. I ooo.

D́eux électrodes supplémentaires placées sur le manomètre $\mathbf{M}$ commandent, par l'intermédiaire d'un relais l'ouverture et la fermeture d'une seconde électrovanne (EV 2) lorsque la chambra respiratoire est en légère surpression. Cette situation se présente parfois au cours des contrôles d'étanchéité, l'installation fonctionnant à vide, lorsque la pression atmosphérique diminue. Cette électrovanne laisse alors s'écouler dans le flacon $\mathrm{D}$ de l'eau située à la base du réservoir $\mathrm{A}$, de façon à augmenter le volume apparent de la chambre respiratoire et à éliminer ainsi la surpression.

\section{F. - Climatisation des chambres respiratoires}

\section{Température.}

Les études que nous réalisons sont effectuées à une température de $2 \mathrm{I} \pm^{\circ}{ }^{\circ} \mathrm{C}$. Les 2 premières chambres respiratoires comportaient une double paroi dans laquelle circulait de l'eau thermostatée ; cette eau baignait également un serpentin en cuivre de $4 \mathrm{~m}$ de longueur placé sur le circuit d'air. La réserve d'eau thermostatée était placée dans un récipient de 50 l ouvert à la partie supérieure et agitée en permanence. Un serpentin en cuivre immergé et alimenté en eau froide du robinet apportait les frigories nécassaires; une résistance électrique de 500 watts immergée était commandée, par l'intermédiaire d'un relais, par un thermomètre à contact placé à l'intérieur de la chambre respiratoire. Cette installation nous donnait satisfaction mais présentait des risques d'accident par défaillance du matériel. 


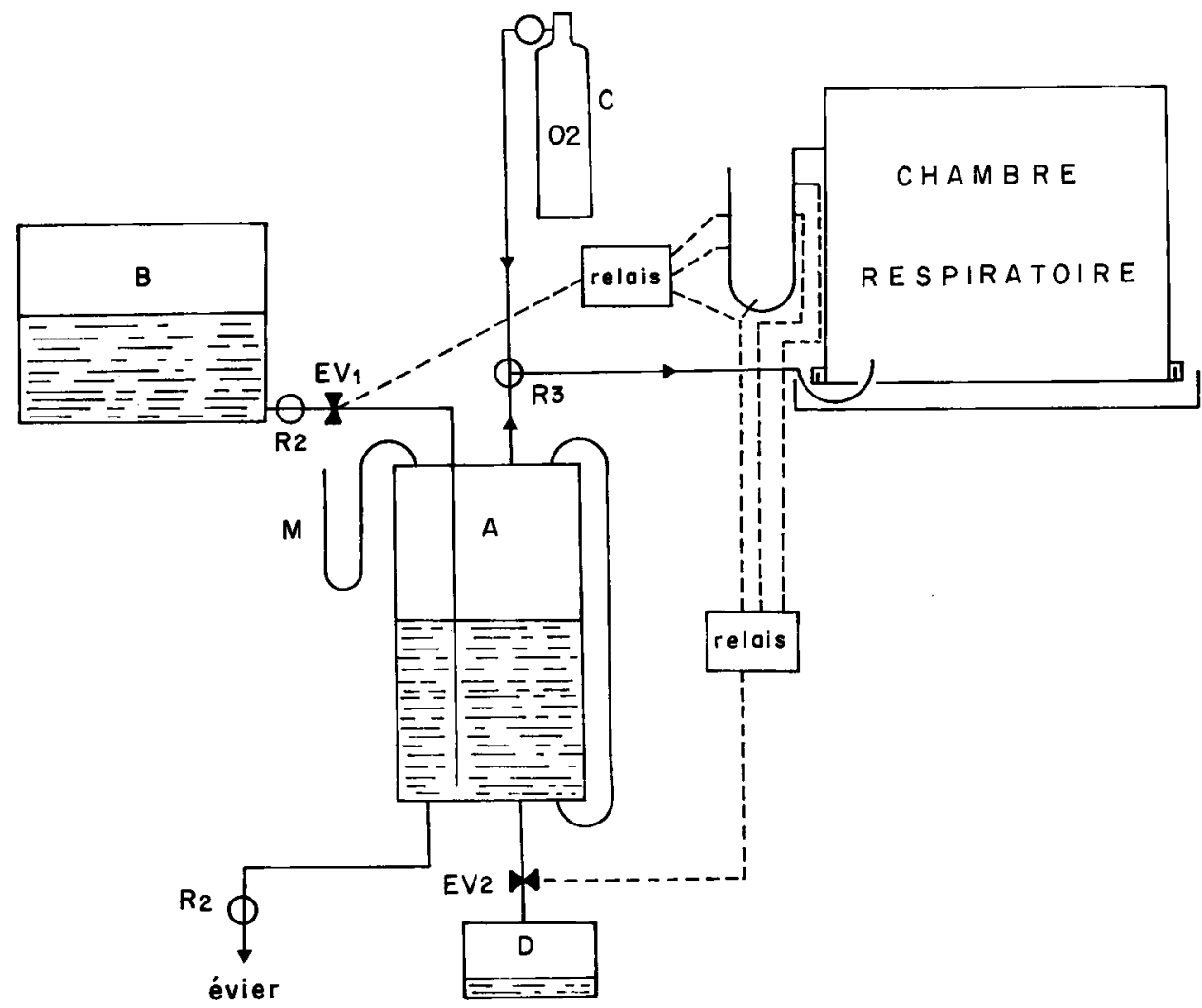

Frg. 4. - Schéma du système d'alimentation automatique en oxygène
$A$ : réservoir d'oxygène
B : réservoir d'eau
C : bouteille d'oxygène
$\mathrm{EV}$ : électrovanne
$\mathrm{D}$ : vase d'expansion
M : manometre
$\mathrm{R}$ : robinet

Design of the automatic oxygen supply system
A : oxygen reservoir
$B$ : water reservoir
C : oxygen cylinder
$\mathrm{D}$ : expansion vessel
EV : solenoid valves
$M$ : manometer
$\mathrm{R}$ : taps

Par la suite, les chambres respiratoires ont été installées dans une pièce conditionnée en température. Il est apparu que notre système de conditionnement était superflu : il suffisait de réduire de $I^{\circ} \mathrm{C}$ la température de la pièce pour avoir la température désirée dans la chambre respiratoire.

\section{Humidité relative.}

En cours de fonctionnement il se crée un équilibre entre les apports de vapeur d'eau, par le joint d'eau, l'animal, l'urine, la nourriture..., et les prélèvements de vapeur d'eau par circulation de l'air dans les absorbeurs. L'humidité relative est comprise entre 60 et $75 \mathrm{p}$. Ioo. On peut la régler, dans une certaine mesure, en faisant varier le débit de l'air à travers les absorbeurs. Cependant nous n'avons pas eu besoin de faire appel à d'autres artifices (joint d'huile à la place du joint d'eau ou réhumidification de l'air avant son retour dans la chambre respiratoire).

Pour le calcul de la quantité d'oxygène consommé nous estimons les variations d'humidité relative à l'aide d'un thermomètre sec et d'un thermomètre humide gradués par $0, \mathrm{I}^{\circ} \mathrm{C}$ mais la vitesse de l'air au niveau de ce dernier étant faible, les valeurs absolues sont fausses par excès, de 5 à ro points. 


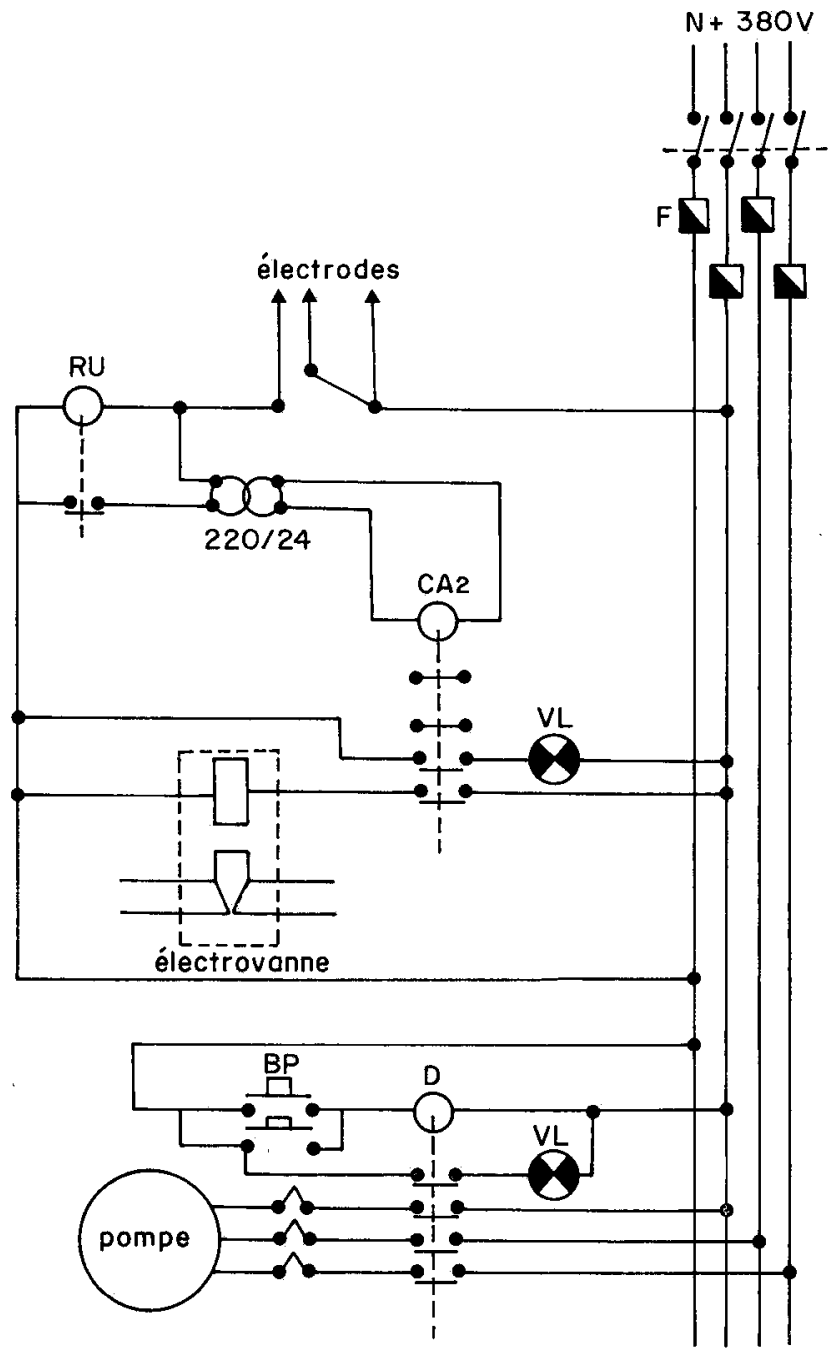

FIG. 5. - Schéma electrique du système d'alimentation automatique en oxygène (alimentation d'une électrovanne)

$\mathrm{F}$ : fusibles

$\mathrm{RU}$ : relais sensible

$\mathrm{CA}_{2}$ : relais de puissance
VL : voyant lumineux BP : bouton poussoir D : discontacteur

Electrical circuit of the automatic oxygen supply system (supply to a solenoid valve)

$F$ : fuses

RU : sensitive relays

$\mathrm{CA}_{2}$ : power relays
VL : signal light

BP : button switches

D : circuit breaker 


\section{III. - CONTRÔLE DU FONCTIONNEMENT}

\section{DES CHAMBRES RESPIRATOIRES}

La meilleure méthode de contrôle de la validité des résultats est la combustion d'une quantité connue d'un produit pur (alcool, propane...). Elle présente des inconvénients pour les installations de grande taille (combustion incomplète du produit, vaporisation) et s'est révélée inapplicable à des chambres respiratoires pour rats en raison des quantités très faibles de produit à faire brûler.

Pour vérifier la validité des mesures des quantités de gaz carbonique produit par le Rat nous réalisons la décomposition progressive d'une quantité connue de carbonate de sodium par une solution d'acide sulfurique dilué. La mesure doit s'effectuer sur une période de 6 heures au moins et la principale difficulté réside dans la décomposition progressive du carbonate; en effet si la décomposition est trop rapide, on risque des fuites par surpression au niveau du joint d'eau et un passage de gaz carbonique dans le spiromètre ou dans le réservoir à oxygène.

A 1'usage nous avons constaté que les principaux risques d'erreurs sont :

- pour le gaz carbonique : une déshydratation incomplète de l'air par l'actigel et la déhydrite suivie d'une captation de traces d'eau par les tamis moléctulaires qui entraîne une erreur par excès ;

— pour l'oxygène : une microfuite sur le circuit de l'air (en général au niveau des absorbeurs ou des tuyaux) compensée par un apport d'oxygène qui provoque une erreur par excès. Ainsi, avec une ventilation de 150 1/heure, une fuite de I/ro ooo correspond à $360 \mathrm{ml} / 24$ heures soit une erreur de 5 à $7 \mathrm{p}$. Ioo.

Nous réservons 2 jours par semaine à ces contrôles en faisant fonctionner les chambres respiratoires à vide. La prise de poids de l'absorbeur de $\mathrm{CO}_{2}$ doit être inférieure à $0,04 \mathrm{~g}$ par 24 heures (soit une erreur relative de 0,2 à $0,3 \mathrm{p}$. roo sur les quantités de $\mathrm{CO}_{2}$ prođuit). Nous avons observé cependant que lorsque les tamis moléculaires régénérés ont été conservés sous vide et sont utilisés pour ces contrôles, leur poids a tendance à augmenter au cours des premières heures puis se stabilise. Ce phénomène est difficile à expliquer et nous avançons l'hypothèse qu'en l'absence de $\mathrm{CO}_{2}$ certains gaz rares pourraient être captés par les sites les plus actifs. Aussi est-il recommandé de faire circuler de l'air déshydraté à travers ces tamis moléculaires avant le début du contrôle.

Les variations de volume corrigé des gaz enfermés dans le système chambre respiratoire, circuit, spiromètre, indiquent la présence de fuites éventuelles. Ces variations doivent être inférieures à $50 \mathrm{ml}$ par 24 heures (soit une erreur de 0,6 à I p. Ioo) et pas systématiquement négatives. La recherche des fuites est délicate en raison de l'impossibilité de créer une surpression dans l'ensemble de l'installation. L'usage d'un détecteur de fréon très sensible s'est révélé inefficace. Enn pratique nous commençons par vérifier l'étanchéité des principaux éléments douteux (absorbeurs, tuyaux) en les immergeant et en les remplissant d'air sous pression. 


\section{IV. - CALCUL DES ÉCHANGES RESPIRATOIRES DU RAT}

Les chambres respiratoires sont arrêtées et ouvertes en moyenne ro minutes par jour. Pour ramener à 24 heures les valeurs mesurées on introduit une correction proportionnelle au temps. Tous les volumes de gaz sont ramenés à zéro ${ }^{\circ} \mathrm{C}, 760 \mathrm{~mm}$ de mercure et secs.

La production de gaz carbonique du rat est la somme de :

- la quantité absorbée par les tamis moléculaires (détermination gravimétrique) ;

- le volume corrigé accumulé dans la chambre respiratoire ;

- éventuellement la correction moyenne observée au cours des contrôles qui précèdent et qui suivent la période de mesure.

Dans le cas de l'utilisation d'un spiromètre ( $\S e \mathrm{I}$ ), la quantité d'oxygène consommé correspond à la somme algébrique de :

- la variation de volume corrigé du spiromètre (Vo ${ }_{s p i}$ );

- la variation de volume corrigé de la chambre respiratoire et du circuit $\left(\mathrm{Vo}_{c h}\right)$;

- la variation de volume correspondant à la variation de surpression dans la chambre respiratoire (Vo $p$ );

- la variation de volume du joint d'eau à l'intérieur de la chambre $\left(\mathrm{Vo}_{j}\right)$;

- le volume corrigé de $\mathrm{CO}_{2}$ accumulé dans la chambre respiratoire $\left(\mathrm{Vo}_{\mathrm{CO}_{2}}\right)$;

- la correction éventuelle observée au cours des contrôles qui précèdent et qui suivent la période de mesure (C) :

$$
\mathrm{O}_{2}=\Delta \mathrm{Vo}_{s p i}+\Delta \mathrm{Vo}_{c h}+\mathrm{Vo}_{\mathrm{CO}_{2}}+\Delta \mathrm{Vo}_{p}+\Delta \mathrm{Vo}_{j}+\mathrm{C}
$$

Les termes $\mathrm{Vo}_{p}$ et $\mathrm{Vo}_{j}$ sont toujours faibles.

Dans le cas de la fourniture automatique d'oxygène par une électrovanne ( $(e$ 2), le calcul est comparable mais la quantité d'oxygène fournie par le réservoir est égale à :

$$
\left(\mathrm{V} \times \mathrm{C}_{i}\right)-\left[\left(\mathrm{V}-\Delta_{p}\right) \times \mathrm{C}_{f}\right]
$$

$\mathrm{V}=$ volume initial apparent d'oxygène dans le réservoir $\mathrm{A}$;

$\Delta_{p}=$ variation de poids $\mathrm{du}$ récipient $\mathrm{B}$;

$\mathrm{C}_{\mathfrak{i}}$ et $\mathrm{C}_{f}=$ coefficients de correction, initial et final, du volume apparent d'oxygène du réservoir.

Pour le calcul de la production de chaleur du rat à partir de ses échanges respiratoires nous utilisons la formule de Brouwer proposée par la F. E. Z. (I964) :

$$
\underset{\mathrm{kcal}}{\mathrm{Q}}=3,866 \mathrm{O}_{2}+\mathrm{I}, 200 \mathrm{CO}_{2}-\mathrm{I}, 43 \mathrm{I} \mathrm{Nu}
$$

Dans le cas où l'aliment comporte des produits particuliers tels que l'acide acétique pour lesquels cette formule donne une valeur différente de la chaleur de combustion, nous corrigeons la production de chaleur totale ainsi calculée en estimant la proportion du produit qui a été catabolisée. 


\section{V. - CONCEPTION ET DÉROULEMENT DES EXPÉRIENCES DE CALORIMÉTRIE INDIRECTE}

Avant le début des mesures les rats reçoivent le régime expérimental et sont placés pendant 4 à 7 jours dans une cage à digestibilité et une chambre d'adaptation comparable à la chambre respiratoire. Les mesures des échanges respiratoires se poursuivent pendant 5 jours en vue d'atténuer les variations quotidiennes et d'améliorer la précision des déterminations. Les 2 jours suivants sont réservés aux contrôles de fonctionnement de l'installation.

Chaque matin la chambre respiratoire est arrêtée pendant Io minutes en moyenne pour effectuer les différentes mesures, alimenter le rat, récolter les refus et les excreta. Les fèces, 1 'urine et les eaux de lavage sont récoltées séparément dans des flacons tarés et conservées à une température de $-I 5^{\circ} \mathrm{C}$. Les récoltes quotidiennes sont cumulées. Un échantillon d'aliment est séché chaque jour à Io0 $\mathrm{C}$ et conservé pour analyse ; les refus sont également séchés à $100^{\circ} \mathrm{C}$.

Compte tenu de la variabilité individuelle observée au niveau des bilans énergétiques et azotés, il est nécessaire de prévoir ro périodes expérimentales par traitement de façon à disposer de 8 résultats valables, au moins, comme le recommande NEHRING (I958). Une étude simple qui comporte 2 régimes et 2 stades physiologiques représente 40 semaines de mesure. Ce nombre montre qu'il est très utile de disposer de 4 chambres respiratoires pour pouvoir réaliser une telle étude dans un délai de 2 à 3 mois. De plus, cet équipement réduit le nombre d'échantillons d'aliments à analyser.

Pour éviter d'avoir à tenir compte du besoin d'entretien des animaux en croissance : nous choisissons des rats de même poids pour les différents lots. Nous préparons 6 à 8 animaux pour disposer de 4 au moins qui présentent le poids voulu et une bonne croissance le jour du début de la mesure ; un petit élevage de rats au laboratoire est à cet égard très utile.

\section{VI. - ÉTABLISSEMENT E'T COMPARAISON DES BILANS ÉNERGÉTIQUES}

Les erreurs expérimentales et analytiques sur les différents termes se répercutent sur les bilans (Graham, Blaxter et Armstrong, 1958). Pour améliorer la précision de nos mesures et contrôler mutuellement nos méthodes nous déterminons la quantité d'énergie fixée par le bilan d'énergie (bilan $Q R$ ) et le bilan $\mathrm{C}-\mathrm{N}$ dont nous rappelons le principe :

$$
\begin{aligned}
\text { Bilan } \mathrm{QR}: & \mathrm{E} \text { fixée }=\mathrm{E} \text { ingérée }-\mathrm{E} \text { fèces }-\mathrm{E} \text { urine }-\mathrm{Q} \\
\text { Bilan } \mathrm{C}-\mathrm{N}: & \mathrm{E} \text { fixée }=\mathrm{E} \text { protéines }+\mathrm{E} \text { lipides } \\
& \mathrm{E} \text { protéines }=\mathrm{N} \text { fixé } \times 6,25 \times 5,7 \\
& \mathrm{E} \text { lipides }=(\mathrm{C} \text { fixé }-\mathrm{C} \text { protéines }) \times \frac{\text { Ioo }}{76,7} \times 9,5
\end{aligned}
$$




\section{Analyse des échantillons}

L'énergie brute est déterminée à l'aide d'un calorimètre adiabatique Gallenkamp étalonné pour une chaleur de combustion de 6,5 kcal. Le dosage est effectué en triple pour les aliments et en double pour les fèces lyophilisées, avec une erreur relative inférieure ou égale à 0,3 p. roo (tabl. I). L'urine est lyophilisée directement dans des sachets en polythène (I50 mg) qui facilitent la combustion (NIJKAMP, I964) et l'erreur relative sur la détermination de la teneur en énergie brute est inférieure ou égale à 3 p. Ioo.

\section{TABLEAU I}

Calcul d'erreur sur les bilans énergétiques et azotés d'un rat de $170 \mathrm{~g}$ pendant une période de 5 jours Calculation of errors in energy and nitrogen balance in a rat of $170 \mathrm{~g}$ during a period of 5 days

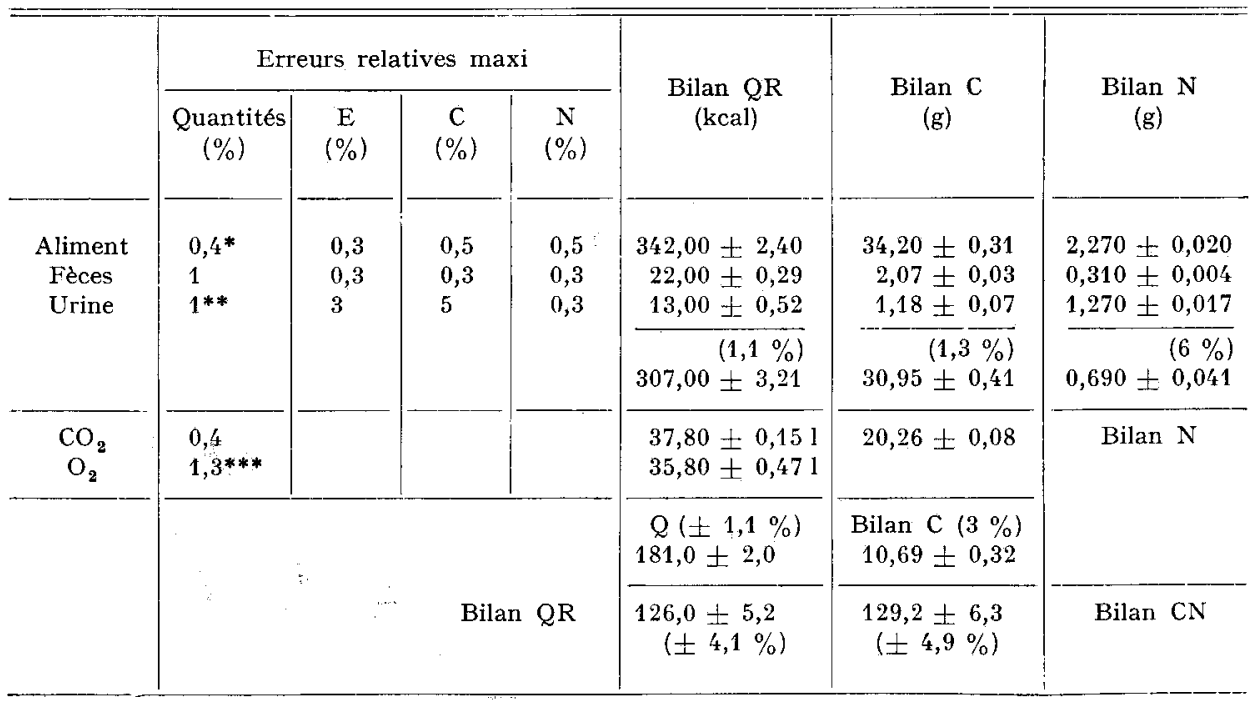

* Gaspillage incontrôlable estimé à 0,1 p. 100 .

** Compte tenu des pertes d'urine au cours des manipulations du rat.

*** Lecture du spiromètre, erreurs sur lectures des températures, pressions, humidité relative. Le bilan azoté ne tient pas compte des pertes de poils et des desquamations difficilement contrôlables.

L'azote total est dosé par la méthode macrokjeldahl avec des prises inférieures à $2 \mathrm{~g}$ de matière sèche, comportant 30 à Ioo $\mathrm{mg}$ d'azote. L'erreur relative est inférieure ou égale à 0,5 p. Ioo pour les aliments (dosage en triple) et 0,3 p. Ioo pour les fèces et l'urine (dosage en double).

La teneur en carbone des aliments et des fèces est déterminée par la méthode de Dumas, selon la technique de VECERA, SNobl et Syné (I958) adaptée au dosage semimicro (prises de $50 \mathrm{mg}$ ). L'échantillon placé dans une nacelle en platine à l'intérieur d'un tube en quartz est brûlé à $700^{\circ} \mathrm{C}$ sous courant d'oxygène. On utilise de l'oxyde cobalto-cobaltique (à $600^{\circ}$ ) comme catalyseur d'oxydation, de la toile d'argent pour capter les halogènes, du bioxyde de manganèse pour piéger les oxydes d'azote et de la déhydrite pour dessécher le gaz vecteur $\left(\mathrm{O}_{2}\right)$. Le gaz carbonique est absorbé par de l'ascarite (amiante sodée) contenue dans 2 absorbeurs placés en série. 
L'échantillon et les 2 absorbeurs sont pesés à l'aide d'une balance de précision (I0o g à $0,05 \mathrm{mg}$ ). L'appareil est étalonné chaque jour avec de la poudre de polythène non hygroscopique. Cette méthode est délicate et longue mais nous donne une précision satisfaisante : erreur maximum de $0,5 \mathrm{p}$. Ioo avec les aliments et $0,3 \mathrm{p}$. Ioo avec les fèces, plus homogènes, comparable à la précision obtenue par NEERGAARD et al. (1969).

Malheureusement cette méthode ne se prête pas au dosage du carbone urinaire en raison des difficultés de combustion. Pour cela, après détermination de l'énergie brute de l'échantillon d'urine lyophilisée, nous vidons la bombe calorimétrique à travers un train d'absorbeurs contenant de la déhydrite, du bioxyde de manganèse

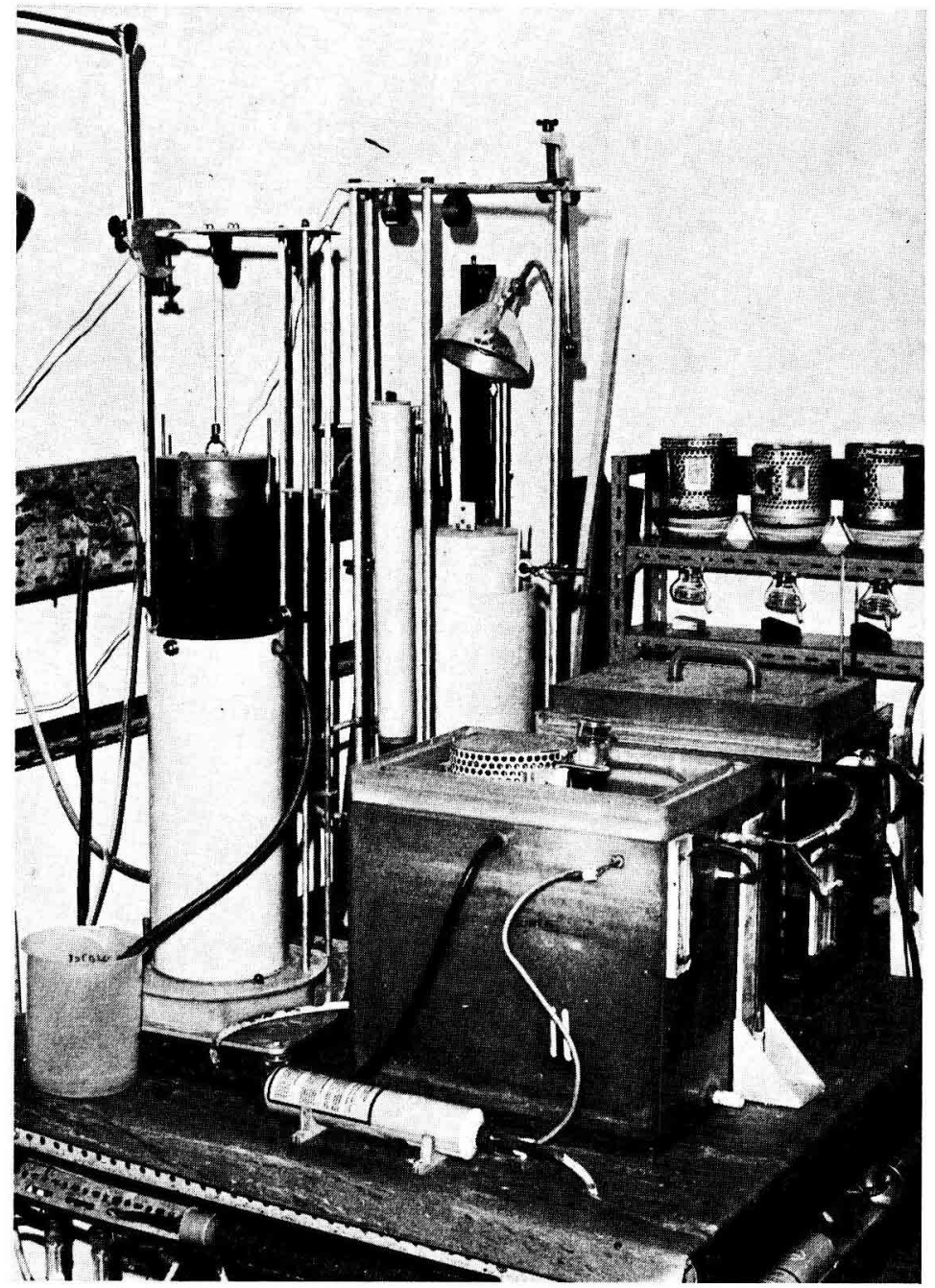

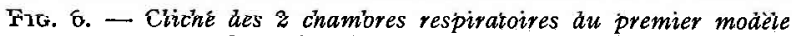
avec les spiromètres et les absorbeurs

Picturs of 2 respiration chambers of the first type with the spirometers and absorption train 
et de l'ascarite (NIJKaMP, I96I) et nous tenons compte du gaz restant dans la bombe. L'erreur maximale admise est de 5 p. Ioo.

La comparaison du bilan $Q R$ et du bilan $\mathrm{C}-\mathrm{N}$ nous permet de contrôler la validité.des résultats des mesures d'oxygène consommé et de gaz.carbonique produit et des différents dosages. Malheureusement les erreurs commises sur les quantités d'aliment ingérées, de fèces et d'urine produites ainsi que sur 1'hydratation des produits séchés ou lyophilisés, se répercutent sur les 2 types de bilan et ne sont pas contrôlables par ce biais. La validité de nos méthodes (VERMOREL et KELILER, I967) peut être jugée satisfaisante puisque la valeur absolue des différences entre les 2 bilans est en moyenne inférieure ou égale à I p. Ioo de l'énergie ingérée et pour l'ensemble d'une expérience les 2 bilans sont en moyenne égaux (BL,AXTER, I967).

\section{VII. - CONCLUSION}

Les chambres respiratoires du type circuit fermé que nous avons réalisées (fig. 6 et 7) permettent d'effectuer des bilans énergétiques et azotés précis pendant des périodes de 24 heures, plusieurs jours consécutifs, avec des rats présentant des

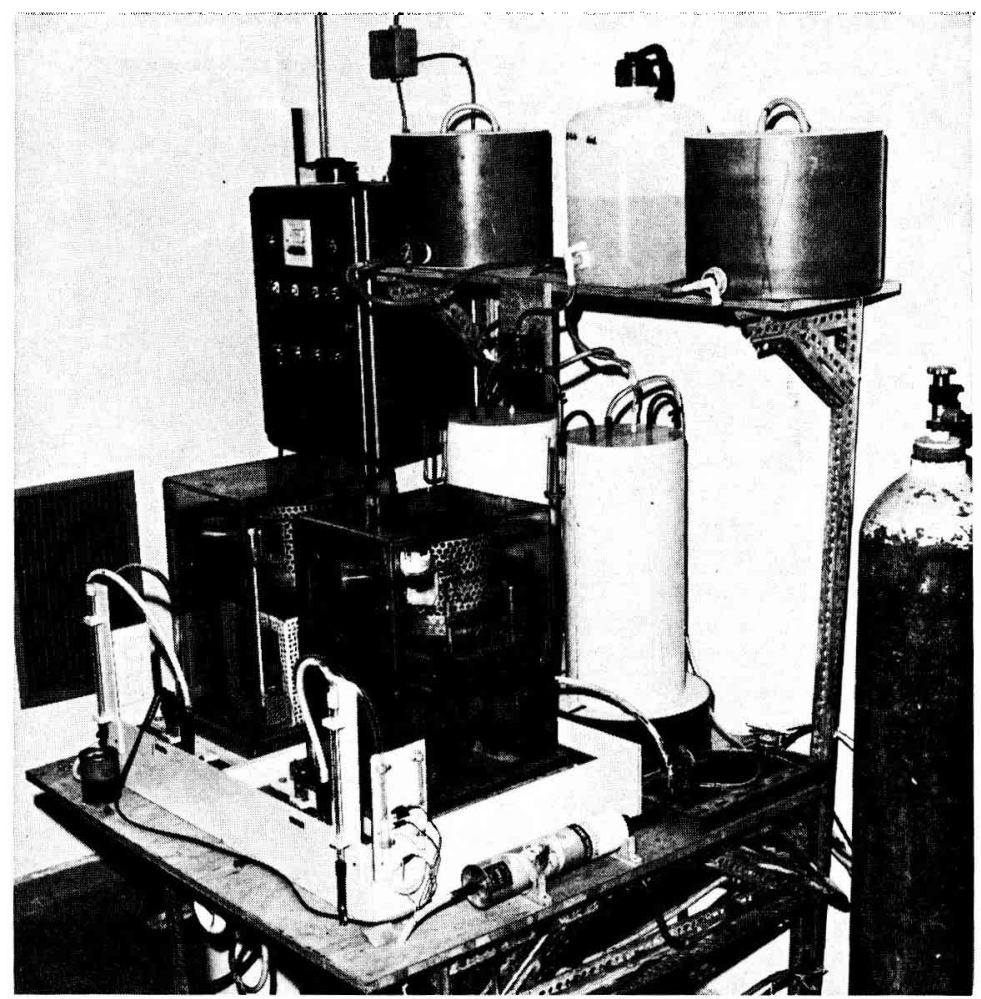

FIG. 7. - Cliché des 2 chambres respiratoires du second modèle avec joint d'eau d̀ la lase et détermination gravimétrique de la quantité d'oxygène consommé

Picture of 2 respiration chambers of the second type with automatic supply device and gravimetric oxygen determination 
vitesses de croissance élevées. Un équipement de 4 chambres nous paraît nécessaire pour conduire d'une façon rationnelle une étude comportant plusieurs traitements, pendant une période de l'ordre de 3 mois, tout en limitant le nombre d'analyses. La précision des résultats obtenus, indiquée à la fois par les analyses, les contrôles effectués sur les chambres respiratoires et la bonne concordance entre le bilan $Q R$ et le bilan $\mathrm{C}-\mathrm{N}$, est satisfaisante mais nécessaire pour les études de nutrition.

Cette installation représente un complément très utile des études en lots par la méthode des abattages comparés. Elle permet en effet, d'étudier pendant de courtes durées la composition du croît des rats ou l'utilisation des nutriments et des régimes à des stades physiologiques (après le sevrage, en fin de croissance ou à l'état adulte) pour lesquels l'intensité de la protéinogenèse et de la lipogenèse et le métabolisme des nutriments sont différents. De plus, il est possible d'étudier la variabilité individuelle des sujets et de suivre, par période de 24 heures ( $\left.{ }^{1}\right)$, leur métabolisme énergétique au cours de restrictions alimentaires ou de réalimentation.

Reçu pour publication en avril 1973.

\section{SUMMARY}

\section{CONSTRUCTION AND OPERATION OF 4 " CIOSED CIRCUIT " RESPIRATION CHAMBERS FOR ENERGY METABOLISM STUDIES IN GROWING RATS}

Four respiration chambers of the "closed circuit " type were designed for precise energy and nitrogen balance studies with growing rats. An original device (figs. I and 2) enables the separation and collection of faeces and urine but involves a significant volume (40 l) of the chambers. The chambers are installed in an air-conditioned room. After drying the air with silica gel and magnesium perchlorate $\mathrm{CO}_{2}$ is absorbed on $5 \AA$ molecular sieves (fig. I) which can be regenerated at $25^{\circ} \mathrm{C}$; the quantity of $\mathrm{CO}_{2}$ produced is determined gravimetrically. Oxygen is supplied either from a precision spirometer or by an automatic supply device (fig. 4 and 5 ) with gravimetric determination. When the rats have been adapted to the experimental procedures and to the respiration chambers, measurements are made over periods of 5 days, with animals of the same weight. The precision of different determinations is shown in table $I$; differences between the $\mathrm{RQ}$ method and $\mathrm{C}$ and $\mathrm{N}$ balance are less than I p. Ioo of energy intake, and, on average are zero.

\section{RÉFÉRENCES BIBLIOGRAPHIQUES}

Blaxter K. L., I967. Techniques in energy metabolism studies and their limitations. Proc. Nutr. Soc., 26, 86-96.

Boiteau H., Debrock C., r96r. Étude de I'élimination de l'anhydride carbonique de l'air au moyen des chaux sodées ou des tamis moléculaires de Linde. Anesth. Anal. Réanimation, 18, I20-I44.

Graham N., Blaxter K. L., Armstrong D. G., 1958. Analytical and other techniques used in respiration calorimetry and their errors. Proc. 1st Symp. Energy Metab. Copenhagen I5-I9 september, pp. I57-I63 E. A. A. P. no 8 . Statens Husdyrbrugsudvalg, Copenhagen.

Neergatad L., Bonsdorff Petersen C., Thorbek G., rg69. Carbon determination in biological materials related to respiration trials. Zeitsch. Tierphysiol. Tierernähr, Futtermittel, 25, 302-308.

(1) Les variations des échanges respiratoires des rats au cours de la journée sont mesurées à l'aide de 2 installations du type " circuit ouvert " : l'animal est placé dans une chambre de volume réduit ( 1 l ou 4 l) balayée par de l'air frais. L'air quittant la chambre traverse les analyseurs de gaz carbonique (o-I p. Ioo) et d'oxygène (20-2I p. I0o); les concentrations de ces gaz sont enregistrées à l'aide de l'installation prévue pour gros animaux (VERMOREL et al., I974). 
Nehring K., 1958. Aufgaben und Arbeiten des Oskar. Kellnet-Institutes für Tierernahrung, Rostock, auf dem Gebiet der Respirations vesuche. Pro. 1st Symp. Energy Metab., Copenhagen 15-19 september. pp. 86-90 E. A. A. P. n०8 Statens Husdyrbrugsudvalg. Copenhagen.

Nijкamp H. J., I96r. Some remarks about the use of the bomb calorimeter. Proc. 2nd Symp. Energy Metab. Wageningen ro-I5 september, p. 86-9o E. A. A. P. no ro. Laboratory of Animal Physiology. Wageningen, Netherlands.

NijкAмP H. J., r964. Some remarks about the determination of the heat of combustion and the carbon content of urine. Proc. 3rd Symp. Energy. Metab., Troon may i964 pp. I47-157; E.A.A.P. no I I. Academic Press, London.

Sch1mmand R., I957. Zur gasanalytischen Untersuchung von Respirationsgasen. Arch. Tierernähr., 7, 98-ro3.

Trussell F., Diehl H., r963. Efficiency of chemical dessicants. Analyt. Chim., 35 B, 674-677.

Vecera M., SNobL D., SYNEK L., 1958. Ein Schnellverfahren zur C-H-Mikro-Bestimmung in organischen Substanzen. Mikrochim. Acta, 9-27.

Vermorel M., Keller J., I967. Utilisation énergétique, par le rat en croissance, des principales céréales composant des régimes isoazotés et équilibrés en acides aminés. Ann. Zootech., 16, 223-234.

Vermorel M., Bouvier J.-C., Bonnet Y., Fauconneau G., I974. Construction et fonctionnement. de 2 chambres respiratoires du type " circuit ouvert" pour jeunes bovins. Ann. Biol. anim. Bioch. Biophys. (sous presse). 